TRANSACTIONS OF THE

AMERICAN MATHEMATICAL SOCIETY

Volume 357, Number 10, Pages 4141-4157

S 0002-9947(04)03654-2

Article electronically published on October 7, 2004

\title{
ON THE FINITE EMBEDDABILITY PROPERTY FOR RESIDUATED ORDERED GROUPOIDS
}

\author{
W. J. BLOK AND C. J. VAN ALTEN
}

\begin{abstract}
The finite embeddability property (FEP) for integral, commutative residuated ordered monoids was established by W. J. Blok and C. J. van Alten in 2002. Using Higman's finite basis theorem for divisibility orders we prove that the assumptions of commutativity and associativity are not required: the classes of integral residuated ordered monoids and integral residuated ordered groupoids have the FEP as well. The same holds for their respective subclasses of (bounded) (semi-)lattice ordered structures. The assumption of integrality cannot be dropped in general - the class of commutative, residuated, lattice ordered monoids does not have the FEP-but the class of $n$-potent commutative residuated lattice ordered monoids does have the FEP, for any $n<\omega$.
\end{abstract}

\section{INTRODUCTION}

The question of whether a given class of algebras has a decidable equational or even universal theory is a natural and important one, in particular, when the class of algebras itself models a logical system. If the class is finitely axiomatizable, one way to establish that its universal theory is decidable is to show that the class has the finite embeddability property (or FEP, for short), i.e., that every finite partial subalgebra of an algebra in the class is isomorphic to a partial subalgebra of a finite algebra in the class. The class of algebraic models of "intuitionistic linear logic with weakening" (consisting of integral, commutative, residuated monoids, and often referred to as integral residuated lattices) has a decidable universal theory (this follows from results by Okada and Terui [16] and Lafont [14]). Indeed, it has the finite embeddability property (see [4]). In this paper we establish the finite embeddability property for the class of integral residuated ordered groupoids and some of its important subclasses, including the one consisting of integral residuated ordered monoids. The members of these classes are of particular importance in the study of logical systems. The most general structures among them arise as models of categorial grammar. The multiplication represents here juxtaposition of parts of speech, and is therefore, in general, neither commutative nor associative (see, for

Received by the editors December 2, 2003.

2000 Mathematics Subject Classification. Primary 06F05; Secondary 03B47, 06F07, 06F99, 08C15, 08A50.

Key words and phrases. Finite embeddability property, residuated ordered groupoid, residuated ordered monoid, residuated lattice, quantale, integrality, finite basis property, divisibility order, well-quasi-order.

Professor Willem Blok tragically passed away shortly after submitting this paper. 
example, [6]). The associative but not necessarily commutative structures figure as the models for non-commutative systems of linear logic.

The finite embeddability property for integral residuated lattices (i.e., for integral, commutative, residuated ordered monoids) was established using Dickson's Lemma (equivalent to what is known as Kripke's Lemma). It is shown here that the proof can be refined so as to apply to the non-commutative or non-associative case if we instead invoke Higman's finite basis theorem for divisibility orders; Dickson's Lemma is a special case of Higman's result.

We refer the reader to [10, [1 and 5] for background on residuated ordered groupoids, semigroups and monoids. The notion of a (residuated) ordered groupoid was first introduced by Birkhoff [1] as a generalization of the (residuated) latticeordered monoids studied by Ward and Dilworth [20. The motivating example for Ward and Dilworth's work was the lattice of ideals of a commutative ring with 1 , endowed with the binary operation of ideal multiplication. The residuation operation is the usual division operation on ideals. The assumption of commutativity was dropped in [7. The multiplication is associative in these examples; a natural example of an, in general, non-associative residuated ordered groupoid is the collection of normal subgroups of a group, partially ordered by inclusion, in which the product of two normal subgroups is defined to be their commutator (see [10]); or, more generally, the collection of congruences of an algebra in a congruence modular variety in which the commutator serves as the groupoid operation. We already commented on the importance of residuated ordered groupoids in the study of logical systems, such as categorial grammars and systems of linear logic and relevance logic; the decidability results we obtain yield corresponding results for the "deducibility problems" for those logics - see [17], [18] and [4] for discussions of this connection, and [2] for the connection between logical systems and classes of algebras in general. Residuated ordered monoids that are complete lattices are known as "quantales", and these have received a considerable amount of attention in the literature (see for example 19]).

The paper is divided into two parts. In the first part we construct, from a partial subalgebra $\boldsymbol{B}$ of a given residuated ordered groupoid $\boldsymbol{A}$, an algebra $\mathbf{D}(\boldsymbol{A}, \boldsymbol{B})$ into which $\boldsymbol{B}$ can be embedded. The constructed algebra $\mathbf{D}(\boldsymbol{A}, \boldsymbol{B})$ is a residuated ordered groupoid that is a complete lattice, and the groupoid operation on $\mathbf{D}(\boldsymbol{A}, \boldsymbol{B})$ distributes over all joins. Moreover, when the groupoid operation of $\boldsymbol{A}$ is commutative or associative, the groupoid operation of $\mathbf{D}(\boldsymbol{A}, \boldsymbol{B})$ is also commutative or associative, respectively. The embedding is also shown to preserve the groupoid identity, if it exists, and all existing meets and joins. In particular, if $\boldsymbol{A}$ is integral (i.e., if it has an identity element and a largest element, which coincide), then $\mathbf{D}(\boldsymbol{A}, \boldsymbol{B})$ is also integral. When the groupoid operation is associative, $\mathbf{D}(\boldsymbol{A}, \boldsymbol{B})$ is, in fact, a "quantale", so the result shows in particular that every residuated ordered monoid can be embedded in a quantale. Our construction owes much to the work on fragments of linear logic in [16] and earlier in [14].

In the second part of the paper we prove that the class IROG of integral residuated ordered groupoids has the FEP. Assuming that $\boldsymbol{B}$ is a finite partial subalgebra of $\boldsymbol{A} \in \mathrm{IROG}$, to prove finiteness of $\mathbf{D}(\boldsymbol{A}, \boldsymbol{B})$, we establish a relationship between elements of $\mathbf{D}(\boldsymbol{A}, \boldsymbol{B})$ and subsets of a finitely generated free groupoid with identity $\mathbf{F}$, ordered by a natural "subword" ordering $\leq^{\mathbf{F}}$. The structure $\langle\mathbf{F}, \leq \mathbf{F}\rangle$ satisfies the requirements of Higman's theorem and hence is well-quasi-ordered, i.e., has no 
infinite antichains or infinite ascending chains. This provides enough information to show $\mathbf{D}(\boldsymbol{A}, \boldsymbol{B})$ is finite and hence that IROG has the FEP. Since $\mathbf{D}(\boldsymbol{A}, \boldsymbol{B})$ is associative whenever $\boldsymbol{A}$ is, it follows immediately that the quasivariety IROM of integral residuated ordered monoids has the FEP as well. We provide an example to show that the assumption of integrality cannot be omitted. We also show that all of these results are preserved as we add various order properties, such as being lattice-ordered or being bounded.

\section{Preliminaries}

Given an algebra $\boldsymbol{A}=\left\langle A,\left\langle f_{i}^{\boldsymbol{A}}: i \in I\right\rangle\right\rangle$ (of any type), and a non-empty subset $B \subseteq A$, the partial subalgebra $\boldsymbol{B}$ of $\boldsymbol{A}$ with domain $B$ is the partial algebra $\left\langle B,\left\langle f_{i}^{\boldsymbol{B}}\right.\right.$ : $i \in I\rangle\rangle$, where for $i \in I, f_{i} k$-ary, $b_{1}, \ldots, b_{k} \in B$,

$$
f_{i}^{\boldsymbol{B}}\left(b_{1}, \ldots, b_{k}\right)= \begin{cases}f_{i}^{\boldsymbol{A}}\left(b_{1}, \ldots, b_{k}\right) & \text { if } f_{i}^{\boldsymbol{A}}\left(b_{1}, \ldots, b_{k}\right) \in B, \\ \text { undefined } & \text { if } f_{i}^{\boldsymbol{A}}\left(b_{1}, \ldots, b_{k}\right) \notin B .\end{cases}
$$

If $\boldsymbol{A}$ is a (partially) ordered algebra $\boldsymbol{A}=\left\langle A,\left\langle f_{i}^{\boldsymbol{A}}: i \in I\right\rangle, \leq^{\boldsymbol{A}}\right\rangle$, with $\leq^{\boldsymbol{A}}$ a partial order on $A$, and $B \subseteq A$ is non-empty, then the partial subalgebra $\boldsymbol{B}$ of $\boldsymbol{A}$ is the ordered partial algebra $\left\langle B,\left\langle f_{i}^{\boldsymbol{B}}: i \in I\right\rangle, \leq^{\boldsymbol{B}}\right\rangle$, where $\leq^{\boldsymbol{B}}=\leq\left.^{\boldsymbol{A}}\right|_{\boldsymbol{B}}$, i.e., for all $a, b \in B, a \leq^{\boldsymbol{B}} b$ iff $a \leq^{\boldsymbol{A}} b$, and where the $f_{i}^{\boldsymbol{B}}$ are defined as before, for $i \in I$.

By an embedding of a partial algebra $\boldsymbol{B}$ into an algebra $\boldsymbol{A}$ we mean a 1-1 map $\iota: B \rightarrow A$ that preserves all existing operations; i.e., if for some operation symbol $f_{i}$ and $b_{1}, \ldots, b_{k} \in B$ we have that $f_{i}^{\boldsymbol{B}}\left(b_{1}, \ldots, b_{k}\right)$ is defined, then $\iota\left(f_{i}^{\boldsymbol{B}}\left(b_{1}, \ldots, b_{k}\right)\right)=$ $f_{i}^{\boldsymbol{A}}\left(\iota\left(b_{1}\right), \ldots, \iota\left(b_{k}\right)\right)$. If $\boldsymbol{B}$ and $\boldsymbol{A}$ are ordered, then $\iota$ is required to satisfy, for $a, b \in B, a \leq^{\boldsymbol{B}} b$ iff $\iota(a) \leq^{\boldsymbol{A}} \iota(b)$.

A class $\mathcal{K}$ of (ordered) algebras has the finite embeddability property (FEP, for short) if every finite partial subalgebra of some member of $\mathcal{K}$ can be embedded into some finite member of $\mathcal{K}$.

From now on in this paper, all classes of algebras considered will be of finite type; i.e., the set of fundamental operations of the algebras considered will be assumed to be finite. Let $\mathcal{K}$ be a class of (ordered) algebras, and suppose that a universal sentence $\varphi$ in the language of $\mathcal{K}$ fails in the class. Then $\varphi$ fails in some $\boldsymbol{A} \in \mathcal{K}$, say. Let $B$ be the set consisting of the elements of $A$ that witness the failure of $\varphi$ as well as all elements of $A$ that occur in the evaluation of terms in $\varphi$. Then $B$ is the universe of a partial subalgebra $\boldsymbol{B}$ of $\boldsymbol{A}$. If the class $\mathcal{K}$ has the FEP, then $\boldsymbol{B}$ is embeddable into a finite algebra $\boldsymbol{C} \in \mathcal{K}$. Clearly, there is a natural interpretation in $\boldsymbol{C}$ for which $\varphi$ fails. Thus, $\varphi$ fails in a finite member of $\mathcal{K}$. If $\mathcal{K}$ is in addition finitely axiomatizable, this allows us to recursively enumerate the universal sentences that fail in $\mathcal{K}$. Under the same hypotheses the universal sentences that hold in $\mathcal{K}$ can be recursively enumerated as well. It follows that if a finitely axiomatizable class of (ordered) algebras has the FEP, then its universal theory is decidable.

If $\mathcal{K}$ is a quasivariety, one has the result below. The proof of (i) $\Rightarrow$ (ii) follows the reasoning of the previous paragraph, and proofs of the implication (ii) $\Rightarrow$ (i) can be found in [9] or [4].

Theorem 1.1. Let $\mathcal{K}$ be a quasivariety of algebras. The following are equivalent.

(i) $\mathcal{K}$ has the FEP,

(ii) $\mathcal{K}$ is generated, as a quasivariety, by its finite members, that is, $\mathcal{K}=I S P P_{U}\left(\mathcal{K}_{F}\right)$, where $\mathcal{K}_{F}$ denotes the class of finite members of $\mathcal{K}$. 
A residuated ordered groupoid is a structure $\boldsymbol{A}=\left\langle A,{ }^{\boldsymbol{A}}, \backslash^{\boldsymbol{A}},\left.\right|^{\boldsymbol{A}}, \leq^{\boldsymbol{A}}\right\rangle$, or less explicitly $\langle A, \cdot, \backslash, /, \leq\rangle$, where the operations $\cdot, \backslash$, and / are binary, $\leq$ is a (partial) order, and for all $a, b, c \in A$

$$
\begin{aligned}
& a \cdot c \leq b \quad \Leftrightarrow \quad c \leq a \backslash b, \\
& c \cdot a \leq b \quad \Leftrightarrow \quad c \leq b / a .
\end{aligned}
$$

The class of all residuated ordered groupoids is denoted ROG. Observe that if $\langle A, \cdot, \backslash, /, \leq\rangle$ is a residuated ordered groupoid, then

$$
\begin{aligned}
& a \backslash b=\operatorname{Max}\{c \in A: a \cdot c \leq b\}, \\
& a / b=\operatorname{Max}\{c \in A: c \cdot b \leq a\} .
\end{aligned}
$$

We shall consider subclasses of ROG that additionally satisfy some of the following properties. We say that a residuated ordered groupoid $\boldsymbol{A}=\langle A, \cdot, \backslash, /, \leq\rangle$ is

- lattice ordered if $\leq$ is a lattice order,

- join-semilattice ordered if $\leq$ is a join-semilattice order,

- meet-semilattice ordered if $\leq$ is a meet-semilattice order,

- lower bounded if $\leq$ has a least element, which we denote by 0 ,

- upper bounded if $\leq$ has a greatest element, which we denote by 1 ,

- bounded if it is both lower and upper bounded,

- associative if - is associative,

- commutative if - is commutative,

- unital if - has an identity element, which we denote by $e$,

- integral if it is upper bounded, and the largest element 1 coincides with the identity $e$ of .

We shall use $\mathcal{P}$ to denote the set consisting of all the properties listed above. Then, for any $\mathcal{Q} \subseteq \mathcal{P}$, we denote by $\mathrm{ROG}^{\mathcal{Q}}$ the class of all residuated ordered groupoids that satisfy each of the properties in $\mathcal{Q}$.

We use the convention that · binds more strongly than other binary connectives and we shall abbreviate $a \cdot b$ by $a b$, etc. We define $x^{0}=e$ and inductively define $x^{k+1}=x\left(x^{k}\right)$ for each $k<\omega$. Note that a residuated ordered groupoid with least element 0 satisfies $0 \leq x \backslash 0$, hence also $x \cdot 0 \leq 0$, so it satisfies $x \cdot 0 \approx 0$. Also, the existence of a least element 0 ensures that a greatest element also exists, namely $1=0 \backslash 0$. Furthermore, by the conditions (1) and (2), commutative residuated ordered groupoids satisfy $x \backslash y \approx y / x$.

A residuated ordered groupoid $\boldsymbol{A}=\langle A, \cdot, \backslash, /, \leq\rangle$ satisfies the following for all $a, b, c, a_{i} \in A, i \in I$ :

$$
\begin{aligned}
& a \leq b \text { implies } a c \leq b c, \\
& a \leq b \text { implies } c a \leq c b, \\
& \text { if } \bigvee_{i \in I} a_{i} \text { exists, then } c\left(\bigvee_{i \in I} a_{i}\right)=\bigvee_{i \in I} c a_{i}, \\
& \text { if } \bigvee_{i \in I} a_{i} \text { exists, then }\left(\bigvee_{i \in I} a_{i}\right) c=\bigvee_{i \in I} a_{i} c .
\end{aligned}
$$

To see that (3) holds, first observe that from $b c \leq b c$ and (2) we get $b \leq b c / c$. If $a \leq b$, then $a \leq b c / c$ hence $a c \leq b c$. The argument for (4) is symmetrical, using the operation $\backslash$ and (19). For (5)), observe that $c a_{i} \leq c\left(\bigvee_{i \in I} a_{i}\right)$ for each $i \in I$ by (4), so $\bigvee_{i \in I} c a_{i} \leq c\left(\bigvee_{i \in I} a_{i}\right)$. Suppose that $c a_{i} \leq d$ for each $i \in I$. Then each $a_{i} \leq c \backslash d$ hence $\bigvee_{i \in I} a_{i} \leq c \backslash d$, so $c\left(\bigvee_{i \in I} a_{i}\right) \leq d$. This proves (56) and the proof of (6) is similar. 
Let $\langle M, \cdot\rangle$ be a groupoid, let $a_{1}, \ldots, a_{n} \in M$ and let $\delta_{1}, \ldots, \delta_{n} \in\{l, r\}$ ( $l$ and $r$ stand for "left" and "right", respectively). We will write $\boldsymbol{a}^{\boldsymbol{\delta}}$ to denote the sequence $a_{1}^{\delta_{1}} \ldots a_{n}^{\delta_{n}}$, we will use $\varepsilon$ to denote the empty sequence and we denote by $M^{l, r}$ the set of all possible $\boldsymbol{a}^{\boldsymbol{\delta}}$, that is,

$$
M^{l, r}=\left\{a_{1}^{\delta_{1}} \ldots a_{n}^{\delta_{n}}: n<\omega ; a_{1}, \ldots, a_{n} \in M ; \delta_{1}, \ldots, \delta_{n} \in\{l, r\}\right\} .
$$

Clearly any two elements of $M^{l, r}$ can be concatenated to form a new element of $M^{l, r}$. The sequence $\boldsymbol{a}^{\boldsymbol{\delta}}$ is to be understood as a unary polynomial operating on $M$, defined inductively as follows: For each $c \in M$, set $\varepsilon(c)=c$ and, for $\boldsymbol{a}^{\boldsymbol{\delta}} \in M^{l, r}$ and $b \in M$, set $\boldsymbol{a}^{\boldsymbol{\delta}} b^{l}(c)=\boldsymbol{a}^{\boldsymbol{\delta}}(b \cdot c)$ and $\boldsymbol{a}^{\boldsymbol{\delta}} b^{r}(c)=\boldsymbol{a}^{\boldsymbol{\delta}}(c \cdot b)$. Thus, for example, $a_{1}^{l} a_{2}^{r} a_{3}^{l}(c)=a_{1} \cdot\left(\left(a_{3} \cdot c\right) \cdot a_{2}\right)$.

The following lemma is an immediate consequence of the above definitions.

Lemma 1.2. Let $\langle M, \cdot\rangle$ be a groupoid ordered by $\leq$ such that (3) -(6) hold. Then, for $\boldsymbol{a}^{\boldsymbol{\delta}} \in M^{l, r}$ and $b, c, c_{i} \in M, i \in I$,

(i) $b \leq c$ implies $\boldsymbol{a}^{\boldsymbol{\delta}}(b) \leq \boldsymbol{a}^{\boldsymbol{\delta}}(c)$, and

(ii) if $\bigvee_{i \in I} c_{i}$ exists, then $\bigvee_{i \in I} \boldsymbol{a}^{\boldsymbol{\delta}}\left(c_{i}\right)$ exists, and $\bigvee_{i \in I} \boldsymbol{a}^{\boldsymbol{\delta}}\left(c_{i}\right)=\boldsymbol{a}^{\boldsymbol{\delta}}\left(\bigvee_{i \in I} c_{i}\right)$.

\section{EMBEDDing THEOREMS}

We shall define a function $\mathbf{D}$ that assigns to a residuated ordered groupoid $\boldsymbol{A}$ and a partial subalgebra $\boldsymbol{B}$ of $\boldsymbol{A}$ a residuated ordered groupoid $\mathbf{D}(\boldsymbol{A}, \boldsymbol{B})$. The algebra $\mathbf{D}(\boldsymbol{A}, \boldsymbol{B})$ is lattice ordered and complete, it contains an isomorphic copy of the partial algebra $\boldsymbol{B}$, and it is obtained from $\boldsymbol{A}$ and $\boldsymbol{B}$ in an economical way.

Let $\boldsymbol{A}=\langle A, \cdot, \backslash, /, \leq\rangle$ be a residuated ordered groupoid and let $\boldsymbol{B}$ be a partial subalgebra of $\boldsymbol{A}$. Let $\langle M, \cdot\rangle$ be the subgroupoid of $\boldsymbol{A}$ generated by $B$. If $X, Y \subseteq M$ and $a \in M$, then $X Y$ denotes $\{a b: a \in X ; b \in Y\}, X a$ denotes $X\{a\}$ and $a X$ denotes $\{a\} X$.

For each $\boldsymbol{a}^{\boldsymbol{\delta}} \in M^{l, r}$ and each $b \in B$, define

$$
\left(\boldsymbol{a}^{\boldsymbol{\delta}}\right)^{-1}(b]=\left\{c \in M: \boldsymbol{a}^{\boldsymbol{\delta}}(c) \leq b\right\}
$$

Note that $\left(\boldsymbol{a}^{\boldsymbol{\delta}}\right)^{-1}(b]$ is a downward closed subset of $M$. For, if $c_{1} \in\left(\boldsymbol{a}^{\boldsymbol{\delta}}\right)^{-1}(b]$ and $c_{2} \leq c_{1}$, then, by Lemma 1.2, $\boldsymbol{a}^{\boldsymbol{\delta}}\left(c_{2}\right) \leq \boldsymbol{a}^{\boldsymbol{\delta}}\left(c_{1}\right) \leq b$ hence $c_{2} \in\left(\boldsymbol{a}^{\boldsymbol{\delta}}\right)^{-1}(b]$. Also note that $\varepsilon^{-1}(b]=\{c \in M: c \leq b\}=(b]^{M}$, the downward closure of $\{b\}$ in $M$. We shall omit the superscript $M$ in the future; (b] will always denote the downward closure in $M$ of the singleton $\{b\}$.

Define

$$
\begin{gathered}
\bar{D}=\left\{\left(\boldsymbol{a}^{\boldsymbol{\delta}}\right)^{-1}(b]: \boldsymbol{a}^{\boldsymbol{\delta}} \in M^{l, r} \text { and } b \in B\right\}, \\
D=\{\bigcap \mathcal{X}: \mathcal{X} \subseteq \bar{D}\} .
\end{gathered}
$$

The collection $D$ of subsets of $M$ is a closure system; the closure operator $C$ over $M$ associated with $D$ is then given by

$$
C(X)=\bigcap\left\{\left(\boldsymbol{a}^{\boldsymbol{\delta}}\right)^{-1}(b] \in \bar{D}: X \subseteq\left(\boldsymbol{a}^{\boldsymbol{\delta}}\right)^{-1}(b]\right\},
$$


for $X \subseteq M$. Define now for all $X, Y \subseteq M$,

$$
\begin{aligned}
X{ }^{D} Y & =C(X Y), \\
X \backslash^{D} Y & =\{a \in M: X a \subseteq Y\}, \\
\left.Y\right|^{D} X & =\{a \in M: a X \subseteq Y\}, \\
\bigwedge_{i \in I}^{D} X_{i} & =\bigcap_{i \in I} X_{i}, \\
\bigvee_{i \in I}^{D} X_{i} & =C\left(\bigcup_{i \in I} X_{i}\right), \\
1^{D} & =M, \\
0^{D} & =\bigcap \bar{D} .
\end{aligned}
$$

If $\boldsymbol{A}$ is unital with identity element $e$, and $e \in B$, then we define

$$
e^{\boldsymbol{D}}=(e]
$$

Lemma 2.1. If $X \subseteq M$ and $Y_{i} \subseteq M$ for $i \in I$, then $X \backslash^{\boldsymbol{D}} \bigcap_{i \in I} Y_{i}=\bigcap_{i \in I}\left(X \backslash^{\boldsymbol{D}} Y_{i}\right)$ and $\left(\bigcap_{i \in I} Y_{i}\right) /{ }^{D} X=\bigcap_{i \in I}\left(Y_{i} /{ }^{\boldsymbol{D}} X\right)$.

Proof. For $a \in M$, we have $X a \subseteq \bigcap_{i \in I} Y_{i}$ iff $X a \subseteq Y_{i}$ for each $i \in I$, from which the first result follows. The second similarly follows from $a X \subseteq \bigcap_{i \in I} Y_{i}$ iff $a X \subseteq Y_{i}$ for each $i \in I$.

Lemma 2.2. If $X \subseteq M$ and $Y \in D$, then $X \backslash^{D} Y \in D$ and $Y /^{\boldsymbol{D}} X \in D$.

Proof. We will show that for $X \subseteq M, \boldsymbol{a}^{\delta} \in M^{l, r}$ and $b \in B$,

$$
\begin{aligned}
& X \backslash^{\boldsymbol{D}}\left(\boldsymbol{a}^{\boldsymbol{\delta}}\right)^{-1}(b]=\bigcap\left\{\left(\boldsymbol{a}^{\boldsymbol{\delta}} c^{l}\right)^{-1}(b]: c \in X\right\}, \\
& \left(\boldsymbol{a}^{\boldsymbol{\delta}}\right)^{-1}(b] /{ }^{\boldsymbol{D}} X=\bigcap\left\{\left(\boldsymbol{a}^{\boldsymbol{\delta}} c^{r}\right)^{-1}(b]: c \in X\right\} .
\end{aligned}
$$

Since $X \subseteq M$, (7) implies that $X \backslash^{\boldsymbol{D}}\left(\boldsymbol{a}^{\boldsymbol{\delta}}\right)^{-1}(b]$ is an intersection of sets that belong to $\bar{D}$, and hence itself belongs to $D$. Since $Y \in D$, we may assume that $Y=$ $\bigcap \mathcal{Y}$ for some $\mathcal{Y} \subseteq \bar{D}$. Then, by the previous lemma, $X \backslash^{D} Y=X \backslash^{D}(\cap \mathcal{Y})=$ $\bigcap\left\{X \backslash^{\boldsymbol{D}}\left(\boldsymbol{a}^{\boldsymbol{\delta}}\right)^{-1}(b]:\left(\boldsymbol{a}^{\boldsymbol{\delta}}\right)^{-1}(b] \in \mathcal{Y}\right\}$, so $X \backslash^{\boldsymbol{D}} Y \in D$ as well.

To see that (7) holds, let $\boldsymbol{a}^{\delta} \in M^{l, r}$ and observe that

$$
\begin{aligned}
& d \in X \backslash^{\mathbf{D}}\left(\boldsymbol{a}^{\boldsymbol{\delta}}\right)^{-1}(b] \\
& \Leftrightarrow \quad X d \subseteq\left(\boldsymbol{a}^{\boldsymbol{\delta}}\right)^{-1}(b] \\
& \Leftrightarrow \quad c d \in\left(\boldsymbol{a}^{\boldsymbol{\delta}}\right)^{-1}(b] \quad \text { for each } c \in X \\
& \Leftrightarrow \boldsymbol{a}^{\boldsymbol{\delta}}(c d) \leq b \quad \text { for each } c \in X \\
& \Leftrightarrow \boldsymbol{a}^{\boldsymbol{\delta}} c^{l}(d) \leq b \quad \text { for each } c \in X \\
& \Leftrightarrow d \in\left(\boldsymbol{a}^{\boldsymbol{\delta}} c^{l}\right)^{-1}(b] \quad \text { for each } c \in X .
\end{aligned}
$$

Similarly, (8) implies that $\left(\boldsymbol{a}^{\boldsymbol{\delta}}\right)^{-1}(b] /{ }^{\boldsymbol{D}} X \in D$, and the proof that $Y /^{\boldsymbol{D}} X \in D$ parallels that given above showing that $X \backslash^{D} Y \in D$. To see that (8) holds, let 


$$
\begin{aligned}
& X \subseteq M, \boldsymbol{a}^{\boldsymbol{\delta}} \in M^{l, r} \text { and } b \in B \text {. Then } \\
& d \in\left(\boldsymbol{a}^{\boldsymbol{\delta}}\right)^{-1}(b] / \boldsymbol{D}_{X} \\
& \Leftrightarrow d X \subseteq\left(\boldsymbol{a}^{\boldsymbol{\delta}}\right)^{-1}(b] \\
& \Leftrightarrow d c \in\left(\boldsymbol{a}^{\boldsymbol{\delta}}\right)^{-1}(b] \text { for each } c \in X \\
& \Leftrightarrow \boldsymbol{a}^{\boldsymbol{\delta}}(d c) \leq b \quad \text { for each } c \in X \\
& \Leftrightarrow \boldsymbol{a}^{\boldsymbol{\delta}} c^{r}(d) \leq b \quad \text { for each } c \in X \\
& \Leftrightarrow d \in\left(\boldsymbol{a}^{\boldsymbol{\delta}} c^{r}\right)^{-1}(b] \text { for each } c \in X .
\end{aligned}
$$

Lemma 2.3. The structure $\mathbf{D}(\boldsymbol{A}, \boldsymbol{B})=\left\langle D, .^{\boldsymbol{D}}, \backslash^{\boldsymbol{D}},\left.\right|^{\boldsymbol{D}}, \subseteq\right\rangle$ is a complete residuated lattice ordered groupoid.

Proof. It is clear that ${ }^{D}$ is a groupoid operation on $D$ and, by Lemma $2.2 D$ is closed under $\backslash^{\boldsymbol{D}}$ and $/^{\boldsymbol{D}}$. It is also clear that $\subseteq$ is a complete lattice order. We need only show that (11) and (22) hold. For $X, Y, Z \in D$, it follows immediately from the definition of $\backslash^{D}$ that $X Z \subseteq Y$ iff $Z \subseteq X \backslash^{D} Y$, but $X{ }^{D} Z \subseteq Y$ iff $X Z \subseteq Y$ since $Y$ is closed with respect to $C$. Thus, (1) holds and (2) follows similarly.

The join and meet operations on $\mathbf{D}(\boldsymbol{A}, \boldsymbol{B})$ with respect to the order given by $\subseteq$, are the operations $\bigvee^{D}$ and $\bigwedge^{D}$ defined earlier. Thus, $1^{\boldsymbol{D}}$ and $0^{\boldsymbol{D}}$ are the greatest and least element of $\mathbf{D}(\boldsymbol{A}, \boldsymbol{B})$, respectively.

Next we show that the construction of $\mathbf{D}(\boldsymbol{A}, \boldsymbol{B})$ preserves various important properties of $\boldsymbol{A}$.

Lemma 2.4. For all $X, Y \subseteq M, C(X) C(Y) \subseteq C(X Y)$.

Proof. Let $X, Y, Z \subseteq M$. By the definition of $\backslash^{D}$ and $/^{D}$, we have the following:

$$
\begin{aligned}
& X Y \subseteq Z \quad \Leftrightarrow \quad Y \subseteq X \backslash^{D} Z, \\
& X Y \subseteq Z \quad \Leftrightarrow \quad X \subseteq Z /^{D} Y .
\end{aligned}
$$

Thus,

$$
\begin{aligned}
& X Y \subseteq C(X Y) \Leftrightarrow Y \subseteq X \backslash^{\boldsymbol{D}} C(X Y) \quad \text { (by (9)) } \\
& \Rightarrow \quad C(Y) \subseteq X \backslash^{D} C(X Y) \quad \text { (by Lemma 2.2) } \\
& \Leftrightarrow X C(Y) \subseteq C(X Y) \quad \text { (by (92)) } \\
& \left.\Leftrightarrow \quad X \subseteq C(X Y) /{ }^{D} C(Y) \quad \text { (by (10) }\right) \\
& \Rightarrow C(X) \subseteq C(X Y) /{ }^{D} C(Y) \quad \text { (by Lemma 2.2) } \\
& \Leftrightarrow C(X) C(Y) \subseteq C(X Y) \quad \text { (by (10) }) \text {. }
\end{aligned}
$$

Lemma 2.5. If $\boldsymbol{A}$ is associative or commutative, then $\mathbf{D}(\boldsymbol{A}, \boldsymbol{B})$ is also associative or commutative, respectively. If $\boldsymbol{A}$ is unital with identity element $e$ and $e \in B$ then $\mathbf{D}(\boldsymbol{A}, \boldsymbol{B})$ is unital with identity element $e^{\boldsymbol{D}}$. If $\boldsymbol{A}$ is integral, then $\mathbf{D}(\boldsymbol{A}, \boldsymbol{B})$ is integral.

Proof. It is clear from the definition of. $\boldsymbol{D}$ that $\mathbf{D}(\boldsymbol{A}, \boldsymbol{B})$ is commutative whenever $\boldsymbol{A}$ is. For the associativity, let $X, Y, Z \in D$. Then $\left(X \cdot{ }^{D} Y\right) \cdot{ }^{\boldsymbol{D}} Z=C(C(X Y) Z)$. By Lemma 2.4 $C(C(X Y) Z)=C(C(X Y) C(Z)) \subseteq C(C((X Y) Z))=C((X Y) Z)$. Since $C$ is a closure operator, $C((X Y) Z) \subseteq C(C(X Y) Z)$, hence $\left(X \cdot{ }^{D} Y\right) \cdot{ }^{D} Z=$ 
$C((X Y) Z)$. Similarly, $X \cdot \boldsymbol{D}(Y \cdot \boldsymbol{D} Z)=C(X(Y Z))$. If $\boldsymbol{A}$ is associative, then $(X Y) Z=X(Y Z)$, so $C((X Y) Z)=C(X(Y Z))$, hence $\mathbf{D}(\boldsymbol{A}, \boldsymbol{B})$ is associative.

Suppose $e$ is an identity element of $\boldsymbol{A}$ and $e \in B$. Recall that $(e]=(\varepsilon)^{-1}(e] \in \bar{D}$. Let $X \in D$. We have $X \subseteq X(e] \subseteq C(X(e])$. Conversely, let $a \in X$ and $b \in(e]$. Since $b \leq e$, we have $a b \leq a e=a$, so $a b \in X$ as $X$ is a downward closed subset of $M$. Thus, $X(e] \subseteq X$, so $C(X(e]) \subseteq X$ and hence $X{ }^{D} \boldsymbol{D}(e]=C(X(e])=X$. Thus, $(e]$ is a right identity element of $\mathbf{D}(\boldsymbol{A}, \boldsymbol{B})$. Similarly, we show that $(e]$ is a left identity element of $\mathbf{D}(\boldsymbol{A}, \boldsymbol{B})$.

If $\boldsymbol{A}$ is integral, then it has a largest element 1 which is also an identity for * Now (1] $=M=1^{\boldsymbol{D}} \in D$. The proof that (1] is an identity element for ${ }^{\boldsymbol{D}}$ is the same as that given for $(e]$ in the paragraph above.

Lemma 2.6. The map $\iota: \boldsymbol{B} \rightarrow \mathbf{D}(\boldsymbol{A}, \boldsymbol{B})$ given by $\iota(b)=(b]$ is an embedding. Existing meets and joins in $\boldsymbol{B}$ are preserved by the embedding. If 1,0 or e exists and belongs to $B$, then $\iota(1)=1^{D}, \iota(0)=0^{D}$ or $\iota(e)=e^{D}$, respectively.

Proof. Let $a, b \in B$ and suppose that $a \backslash b \in B$. We need to show that $\iota(a \backslash b)=$ $\iota(a) \backslash \boldsymbol{D}_{\iota}(b)$, i.e., $(a \backslash b]=(a] \backslash^{\boldsymbol{D}}(b]$, or

$$
\{c \in M: a c \leq b\}=\{c \in M:(a] c \subseteq(b]\} .
$$

Let $c \in M$ with $a c \leq b$, and let $d \leq a$. By (3), $d c \leq a c \leq b$, hence $d c \in(b]$, as required. Conversely, if $(a] c \subseteq(b]$, then $a c \leq b$. When $b / a \in B$, the proof that $(b / a]=(b] / \boldsymbol{D}(a]$ is similar.

Suppose $a b \in B$. We need to show that $\iota(a \cdot b)=\iota(a) \cdot{ }^{D} \iota(b)$, i.e., $(a b]=C((a](b])$. Since $a b \in(a](b] \subseteq C((a](b])$, which is downward closed, we get $(a b] \subseteq C((a](b])$. Conversely, let $c \leq a$ and $d \leq b$. Then $c d \leq a b$ by (3) and (4) , so $(a](b] \subseteq(a b]$, hence $C((a](b]) \subseteq(a b]$.

We have shown that $\iota$ preserves $\backslash, /$, and . when these operations are defined. Clearly $\iota$ also preserves the order, as for $a, b \in B$, we have $a \leq b$ if and only if $\iota(a)=(a] \subseteq(b]=\iota(b)$.

To see that $\iota$ preserves existing meets and joins, let $c_{i} \in B$ for each $i \in I$ and first suppose that $\bigwedge_{i \in I} c_{i}$ exists in $\boldsymbol{A}$ and that $\bigwedge_{i \in I} c_{i} \in B$. It is easy to see that $\iota\left(\bigwedge_{i \in I} c_{i}\right)=\left(\bigwedge_{i \in I} c_{i}\right]=\bigcap_{i \in I}\left(c_{i}\right]=\bigwedge_{i \in I}^{D}\left(c_{i}\right]=\bigwedge_{i \in I}^{D} \iota\left(c_{i}\right)$. Next suppose $\bigvee_{i \in I} c_{i}$ exists in $\boldsymbol{A}$ and that $\bigvee_{i \in I} c_{i} \in B$. We need to show that $\iota\left(\bigvee_{i \in I} c_{i}\right)=\bigvee_{i \in I}^{D} \iota\left(c_{i}\right)$, i.e., $\left(\bigvee_{i \in I} c_{i}\right]=C\left(\bigcup_{i \in I}\left(c_{i}\right]\right)$. Clearly, each $\left(c_{i}\right]$ is contained in $\left(\bigvee_{i \in I} c_{i}\right]$, hence $C\left(\bigcup_{i \in I}\left(c_{i}\right]\right) \subseteq\left(\bigvee_{i \in I} c_{i}\right]$. Conversely, let $\left(\boldsymbol{a}^{\boldsymbol{\delta}}\right)^{-1}(b] \in \bar{D}$ such that $\bigcup_{i \in I}\left(c_{i}\right] \subseteq$ $\left(\boldsymbol{a}^{\boldsymbol{\delta}}\right)^{-1}(b]$. Then $c_{i} \in\left(\boldsymbol{a}^{\boldsymbol{\delta}}\right)^{-1}(b]$, and hence $\boldsymbol{a}^{\boldsymbol{\delta}}\left(c_{i}\right) \leq b$, for each $i \in I$. By Lemma 1.2 $\boldsymbol{a}^{\boldsymbol{\delta}}\left(\bigvee_{i \in I} c_{i}\right)=\bigvee_{i \in I} \boldsymbol{a}^{\boldsymbol{\delta}}\left(c_{i}\right) \leq b$, hence $\bigvee_{i \in I} c_{i} \in\left(\boldsymbol{a}^{\boldsymbol{\delta}}\right)^{-1}(b]$. Thus

$$
\bigvee_{i \in I} c_{i} \in \bigcap\left\{\left(\boldsymbol{a}^{\boldsymbol{\delta}}\right)^{-1}(b] \in \bar{D}: \bigcup_{i \in I}\left(c_{i}\right] \subseteq\left(\boldsymbol{a}^{\boldsymbol{\delta}}\right)^{-1}(b]\right\}=C\left(\bigcup_{i \in I}\left(c_{i}\right]\right),
$$

so $\left(\bigvee_{i \in I} c_{i}\right] \subseteq C\left(\bigcup_{i \in I}\left(c_{i}\right]\right)$.

Suppose $\boldsymbol{A}$ has a greatest element 1 and that $1 \in B$. Then (1] $=M=1^{\boldsymbol{D}}$, as required. Suppose $\boldsymbol{A}$ has a least element 0 . We have already seen that then $c 0=0 c=0$, for all $c \in M$. It follows that for any $\boldsymbol{a}^{\boldsymbol{\delta}} \in M^{l, r}$ and $b \in B$, $\boldsymbol{a}^{\boldsymbol{\delta}}(0)=0 \leq b$, so $0 \in\left(\boldsymbol{a}^{\boldsymbol{\delta}}\right)^{-1}(b]$. Thus $0 \in \bigcap \bar{D}$, so $(0]=\{0\} \subseteq \bigcap \bar{D} \subseteq(0]$ and hence $(0]=\bigcap \bar{D}=0^{D}$. Finally, if $\boldsymbol{A}$ has an identity element $e$ and $e \in B$, then $(e]=e^{\boldsymbol{D}}$ by definition. 
Collecting the previous results, we obtain

Theorem 2.7. If $\boldsymbol{A}$ is a residuated ordered groupoid and $\boldsymbol{B}$ is a partial subalgebra of $\boldsymbol{A}$, then $\iota$ is an embedding of $\boldsymbol{B}$ into the residuated, complete lattice ordered groupoid $\mathbf{D}(\boldsymbol{A}, \boldsymbol{B})$. If $\boldsymbol{A} \in \mathrm{ROG}^{\mathcal{Q}}$ for any $\mathcal{Q} \subseteq \mathcal{P}$, then $\mathbf{D}(\boldsymbol{A}, \boldsymbol{B}) \in \mathrm{ROG}^{\mathcal{Q}}$, and $\iota$ preserves any meets, joins, greatest and smallest element, and the identity of $\boldsymbol{A}$ that exist in $\boldsymbol{B}$.

If, in the above theorem, we take for our partial subalgebra of $\boldsymbol{A}$ the algebra $\boldsymbol{A}$ itself, we obtain an embedding of $\boldsymbol{A}$ into $\mathbf{D}(\boldsymbol{A}, \boldsymbol{A})$. This yields

Corollary 2.8. Every residuated ordered groupoid can be embedded into a residuated, complete lattice ordered groupoid. For $\mathcal{Q} \subseteq \mathcal{P}$, if $\boldsymbol{A} \in \mathrm{ROG}^{\mathcal{Q}}$, then $\boldsymbol{A}$ can be embedded into a complete lattice ordered member of $\mathrm{ROG}^{\mathcal{Q}}$, and the embedding preserves any meets, joins, greatest and smallest element, and the identity, provided they exist in $\boldsymbol{A}$.

\section{THE FINITE EMBEDDABILITY PROPERTY}

We will now establish the FEP for various classes of integral residuated ordered groupoids, i.e., of residuated ordered groupoids with a greatest element 1 that at the same time serves as the identity for the groupoid operation. In the next section we will see that, in general, the assumption of integrality cannot be omitted.

We use IROG to denote the class of all integral residuated ordered groupoids. For each $\mathcal{Q} \subseteq \mathcal{P}$, let IROG ${ }^{\mathcal{Q}}$ denote the subclass of IROG that satisfies the properties in $\mathcal{Q}$.

The class IROG satisfies

$$
x \leq y \Leftrightarrow x 1 \leq y \quad \Leftrightarrow 1 \leq x \backslash y \Leftrightarrow 1 \approx x \backslash y .
$$

Thus, $\leq$ is equationally definable in terms of 1 and $\backslash$, so we may replace $\leq$ by 1 in the language of IROG.

Consider the following identities and quasi-identities:

$$
\begin{aligned}
& x 1 \approx 1 x \approx x, \\
& x \backslash x \approx 1, \\
& x \backslash y \approx 1 \quad \& \quad y \backslash x \approx 1 \quad \Rightarrow \quad x \approx y, \\
& x \backslash y \approx 1 \quad \& \quad y \backslash z \approx 1 \quad \Rightarrow \quad x \backslash z \approx 1, \\
& x \backslash 1 \approx 1, \quad \Rightarrow \quad z \backslash(x \backslash y) \approx 1, \\
& x z \backslash y \approx 1 \quad \Rightarrow \quad x z \backslash y \approx 1, \\
& z \backslash(x \backslash y) \approx 1 \quad \Rightarrow \quad z \backslash(y / x) \approx 1, \\
& z x \backslash y \approx 1 \quad \Rightarrow \quad z x \backslash y \approx 1 .
\end{aligned}
$$

Condition (11) states that 1 is the identity element, (12), (13) and (14) that $\leq$ (defined by $x \leq y$ iff $x \backslash y=1$ ) is a partial order and (15) that 1 is the greatest element. The conditions (16)-(19) are equivalent to the residuation properties (10) and (2). It is clear that IROG is axiomatized by (11)-(19), and therefore IROG is a quasivariety, so Theorem 1.1 holds for IROG. There does not exist an equational axiomatization of IROG, i.e., IROG is not a variety. Higgs [13] exhibited a (commutative and associative) algebra in IROG that has a homomorphic image which is not in IROG. 
If $\boldsymbol{B}$ is a partial subalgebra of $\boldsymbol{A} \in \mathrm{IROG}$, then, by Theorem 2.7 the algebra $\mathbf{D}(\boldsymbol{A}, \boldsymbol{B})$ is in IROG and $\boldsymbol{B}$ is embeddable into $\mathbf{D}(\boldsymbol{A}, \boldsymbol{B})$. We shall show that, when $\boldsymbol{B}$ is finite, so is $\mathbf{D}(\boldsymbol{A}, \boldsymbol{B})$, and hence that IROG has the FEP. We need the following definitions and lemmas.

Let $\mathbf{F}(k)$ be the free groupoid with identity 1 on $k$ generators $x_{1}, \ldots, x_{k}$. Each element of $\mathrm{F}(k)$ is assumed to be reduced; that is, apart from the identity element itself, no element contains any occurrences of 1 . We shall abbreviate $s \cdot \mathbf{F} t$ by $s t$, for all $s, t \in \mathrm{F}(k)$. Define a relation on $\mathrm{F}(k)$ by $s \leq \mathbf{F} t$ iff some instances of variables in $s$ can be replaced by 1's in such a way that it reduces to $t$ (by repeated application of the identities $x 1 \approx x$ and $1 x \approx x)$. It is clear that $\leq \mathbf{F}$ is reflexive and transitive. If $s \leq^{\mathbf{F}} t$, then either $s=t$ or $t$, has a lower degree of complexity than $s$. Thus, if we also have $t \leq \mathbf{F} s$, it must follow that $s=t$, so $\leq^{\mathbf{F}}$ is a partial order. Evidently, $s \leq \mathbf{F} t$ implies $s u \leq \mathbf{F}$ tu and $u s \leq \mathbf{F} u t$, hence $\langle\mathrm{F}(k), \cdot, \leq \mathbf{F}\rangle$ is an ordered groupoid. It is integral, since $1 \in \mathrm{F}(k)$ is the identity element for $\cdot$, and $s \leq \mathbf{F} 1$, for all $s \in \mathrm{F}(k)$. We will now show that it is residuated.

Observe that the only nontrivial identities satisfied by the free goupoid with identity are $x 1 \approx x$ and $1 x \approx x$. Thus if $s, t, u, v$ are elements of $\mathrm{F}(k)$ and all different from the identity, and $s u=t v$, then $s=t$ and $u=v$.

Lemma 3.1. Let $s, u, t, v \in \mathrm{F}(k)$ with $t \neq 1$ and $v \neq 1$. If $s u \leq \mathbf{F}$ tv, then one of the following must hold:

(i) $s \leq \mathbf{F} t v$,

(ii) $u \leq \mathbf{F} t v$,

(iii) $s \leq \mathbf{F}$ t and $u \leq \mathbf{F} v$.

Proof. We can replace instances of the free variables in $s u$ by 1 's in such a way that it reduces to $t v$. Let $s^{\prime}$ and $u^{\prime}$ be the resulting reductions of $s$ and $u$, respectively, so $s^{\prime} u^{\prime}=t v$. If $s^{\prime}=1$, then $u \leq \mathbf{F} t v$. Similarly, if $u^{\prime}=1$, then $s \leq \mathbf{F} t v$. If $s^{\prime} \neq 1$ and $u^{\prime} \neq 1$, then, since we are in a free groupoid with identity, we have $s^{\prime}=t$ and $u^{\prime}=v$, hence $s \leq^{\mathbf{F}} t$ and $u \leq \mathbf{F} v$.

For $s, t \in \mathrm{F}(k)$ define

$$
\begin{aligned}
s \backslash{ }^{\mathbf{F}} t & =\operatorname{Max}\{u \in \mathrm{F}(k): s u \leq \mathbf{F} t\}, \\
t /{ }^{\mathbf{F}} s & =\operatorname{Max}\{u \in \mathrm{F}(k): u s \leq \mathbf{F} t\} .
\end{aligned}
$$

In the following lemma we shall see that $\backslash^{\mathbf{F}}$ and $/ \mathbf{F}$ are well-defined binary operations on $\mathrm{F}(k)$.

Lemma 3.2. The structure $\langle\mathrm{F}(k), \cdot \mathbf{F}, \backslash \mathbf{F}, / \mathbf{F}, \leq \mathbf{F}\rangle$ is an integral residuated ordered groupoid.

Proof. In order to show that $\left\langle\mathrm{F}(k),{ }^{\mathbf{F}}, \backslash \mathbf{F}, / \mathbf{F}, \leq^{\mathbf{F}}\right\rangle$ is a residuated ordered groupoid it suffices to prove that the residuation conditions (11) and (2) hold. Let $s, t \in \mathrm{F}(k)$.

(i) Suppose $t$ is a variable, say $t=x_{1}$. Then $s \backslash^{\mathbf{F}} x_{1}=1$ if $x_{1}$ occurs in $s$ and $s \backslash \mathbf{F} x_{1}=x_{1}$ if $x_{1}$ does not occur in $s$. In both cases $s u \leq{ }^{\mathbf{F}} t$ iff $u \leq \mathbf{F} s \backslash t$.

(ii) Next, suppose that $t=t_{1} t_{2}$ with $t_{1} \neq 1$ and $t_{2} \neq 1$. If $s \leq^{\mathbf{F}} t$, then $s \backslash^{\mathbf{F}} t=1$. If $s \bigsqcup^{\mathbf{F}} t$ but $s \leq^{\mathbf{F}} t_{1}$, then $\left.s\right\rangle^{\mathbf{F}} t=t_{2}$. To show this, suppose first that $u \leq t_{2}$. Then $s u \leq t_{1} t_{2}=t$. Conversely, suppose $s u \leq^{\mathbf{F}} t=t_{1} t_{2}$. By Lemma 3.1 either $s \leq \mathbf{F} t$, but we have assumed otherwise, or $u \leq \mathbf{F} t$, hence also $u \leq \mathbf{F} t_{2}$, or $s \leq \mathbf{F} t_{1}$ and $u \leq{ }^{\mathbf{F}} t_{2}$. Thus, $u \leq^{\mathbf{F}} t_{2}$ in each case. 
Finally, if $s \not^{\mathbf{F}} t$ and $s \not^{\mathbf{F}} t_{1}$, then $s \backslash \mathbf{F} t=t$. For by Lemma 3.1] if $s u \leq^{\mathbf{F}} t$ then either $s \leq^{\mathbf{F}} t$ or $u \leq^{\mathbf{F}} t$ or both $s \leq^{\mathbf{F}} t_{1}$ and $u \leq \mathbf{F} t_{2}$. The only possibility is $u \leq \mathbf{F} t$. Conversely, if $u \leq \mathbf{F} t$, then $s u \leq^{\mathbf{F}} t$ as well.

Together (i) and (ii) show that $\backslash \mathbf{F}$ is the residual of $\cdot \mathbf{F}$ with respect to $\leq \mathbf{F}$, i.e., that the residuation condition (1) holds. Condition (2) is proved similarly. Thus $\left\langle\mathrm{F}(k),{ }^{\mathbf{F}}, \backslash \mathbf{F}, / \mathbf{F}, \leq^{\mathbf{F}}\right\rangle$ is a residuated ordered groupoid. It is integral since 1 is both the greatest element of $\leq^{\mathbf{F}}$ and an identity for $\cdot \mathbf{F}$.

Recall that $\mathrm{F}(k)^{l, r}$ is the set of all sequences $\boldsymbol{u}^{\boldsymbol{\delta}}=u_{1}^{\delta_{1}} \ldots u_{n}^{\delta_{n}}$, where $n<\omega$, $u_{i} \in \mathrm{F}(k)$ and $\delta_{i} \in\{l, r\}$ for $i \in\{1, \ldots, n\}$, and that each $\boldsymbol{u}^{\delta} \in \mathrm{F}(k)^{l, r}$ can be viewed as a unary polynomial over the language $\{\cdot\}$ acting on $\mathrm{F}(k)$. We will now assign to each such polynomial $\boldsymbol{u}^{\boldsymbol{\delta}}$ a new unary polynomial $\rho\left(\boldsymbol{u}^{\boldsymbol{\delta}}\right)$ over the language $\{\backslash, /\}$ inductively as follows. For the empty sequence, set $\rho(\varepsilon)(x)=x$. If $\rho\left(\boldsymbol{u}^{\boldsymbol{\delta}}\right)(x)$ has been defined, then, for $v \in \mathrm{F}(k)$, define $\rho\left(\boldsymbol{u}^{\boldsymbol{\delta}} v^{l}\right)(x)=v \backslash \mathbf{F}\left(\rho\left(\boldsymbol{u}^{\boldsymbol{\delta}}\right)(x)\right)$ and $\rho\left(\boldsymbol{u}^{\boldsymbol{\delta}} v^{r}\right)(x)=\left(\rho\left(\boldsymbol{u}^{\boldsymbol{\delta}}\right)(x)\right) /{ }^{\mathbf{F}} v$. To illustrate the definition, $\rho\left(a_{1}^{l} a_{2}^{r} a_{3}^{l}\right)(x)=$ $a_{3} \backslash \mathbf{F}\left(\left(a_{1} \backslash \mathbf{F} x\right) / \mathbf{F}_{a_{2}}\right)$.

Lemma 3.3. For each $\boldsymbol{u}^{\boldsymbol{\delta}} \in \mathrm{F}(k)^{l, r}$ and all $s, t \in \mathrm{F}(k)$,

(i) $\boldsymbol{u}^{\boldsymbol{\delta}}(s) \leq t \quad \Leftrightarrow \quad s \leq \rho\left(\boldsymbol{u}^{\boldsymbol{\delta}}\right)(t)$,

(ii) $t \leq \mathbf{F} \rho\left(\boldsymbol{u}^{\boldsymbol{\delta}}\right)(t)$.

Proof. (i) The proof is by induction on the length of a sequence $\boldsymbol{u}^{\boldsymbol{\delta}} \in \mathrm{F}(k)^{l, r}$. Clearly, for the empty sequence we have $\varepsilon(s) \leq t$ iff $s \leq t$ iff $s \leq \rho(\varepsilon)(t)$. Next consider a sequence $\boldsymbol{u}^{\boldsymbol{\delta}} v^{l}$, where $\boldsymbol{u}^{\boldsymbol{\delta}} \in \mathrm{F}(k)^{l, r}$, and $v \in \mathrm{F}(k)$. Then

$$
\begin{aligned}
\boldsymbol{u}^{\boldsymbol{\delta}} v^{l}(s) \leq t & \Leftrightarrow \boldsymbol{u}^{\boldsymbol{\delta}}(v s) \leq t \\
& \Leftrightarrow v s \leq \rho\left(\boldsymbol{u}^{\boldsymbol{\delta}}\right)(t) \\
& \Leftrightarrow s \leq v \backslash \mathbf{F} \rho\left(\boldsymbol{u}^{\boldsymbol{\delta}}\right)(t) \\
& \Leftrightarrow s \leq \rho\left(\boldsymbol{u}^{\boldsymbol{\delta}} v^{l}\right)(t) .
\end{aligned}
$$

A similar argument applies to a sequence of the form $\boldsymbol{u}^{\boldsymbol{\delta}} v^{r}$.

(ii) Note that for $s, t \in \mathrm{F}(k)$, we have $s t \leq \mathbf{F} s$ and $s t \leq \mathbf{F} t$ by integrality. It follows that for all $\boldsymbol{u}^{\boldsymbol{\delta}} \in \mathrm{F}(k)^{l, r}$ and $t \in \overline{\mathrm{F}}(k)$ we have $\boldsymbol{u}^{\boldsymbol{\delta}}(t) \leq \mathbf{F} t$. Then $t \leq \mathbf{F} \rho\left(\boldsymbol{u}^{\boldsymbol{\delta}}\right)(t)$ follows by (i).

We rephrase some definitions and a result by Higman from 12] to suit our needs. Given a set $A$ with quasi-order $\preceq$, we say that $\langle A, \preceq\rangle$ has the finite basis property if every downward closed subset of $A$ is the downward closure of a finite set. Given an algebra $\boldsymbol{A}$ (of any type), a quasi-order $\preceq$ on $A$ is called a divisibility order if it satisfies the following conditions:

(i) Each operation of $\boldsymbol{A}$ preserves the quasi-order in each of its arguments.

(ii) If $f^{\boldsymbol{A}}$ is an $n$-ary operation of $\boldsymbol{A}$ and $a_{1}, \ldots, a_{n} \in A$, then $f^{\boldsymbol{A}}\left(a_{1}, \ldots, a_{n}\right)$ $\preceq a_{i}$ for each $i=1, \ldots, n$.

Theorem 3.4 (12, Theorem 1.2). Let $\boldsymbol{A}$ be an algebra of finite type endowed with a divisibility order $\preceq$. If $\boldsymbol{A}$ is generated by a set $S \subseteq A$, and $\langle S, \preceq \mid s\rangle$ has the finite basis property, then $\langle A, \preceq\rangle$ has the finite basis property.

It is well-known that a quasi-ordered set $\langle A, \preceq\rangle$ has the finite basis property if and only if it is "well-quasi-ordered", i.e., if and only if it possesses no infinite antichains and no infinite ascending chains. We refer the reader to [21] for a full discussion of well-quasi-orders. 
Corollary 3.5. The ordered set $\left\langle\mathrm{F}(k), \leq^{\mathbf{F}}\right\rangle$ is well-quasi-ordered.

Proof. We first show that $\leq \mathbf{F}$ is a divisibility order on $\mathbf{F}(k)$. The algebra $\mathbf{F}(k)$ has only one (nonconstant) operation, namely its groupoid operation. $\mathbf{F}$. We have already observed that. $\mathbf{F}$ is order-preserving in both of its arguments, so (i) holds. We have also seen that, by integrality, $s t \leq \mathbf{F} s$ and $s t \leq \mathbf{F} t$, for all $s, t \in \mathrm{F}(k)$, so (ii) holds as well.

The set $S=\left\{x_{1}, \ldots, x_{k}\right\}$ is a generating set for $\mathbf{F}(k)$. Hence, in view of Theorem 3.4, in order to show that $\langle\mathrm{F}(k), \leq \mathbf{F}\rangle$ has the finite basis property, it suffices to observe that $\left\langle S, \leq \mathbf{F} \mid{ }_{S}\right\rangle$ has the finite basis property. This is trivial since any finite quasi-ordered set has the finite basis property.

We will apply Corollary 3.5 in the following way. For any $X \subseteq \mathrm{F}(k)$, we write $(X]^{\mathbf{F}}$ for the downward closure of $X$ in $\left\langle\mathrm{F}(k), \leq^{\mathbf{F}}\right\rangle$ and Max $X$ for the set of maximal elements of $X$. Since $X$ contains no infinite ascending chains, $(X]^{\mathbf{F}}=(\operatorname{Max} X]^{\mathbf{F}}$. The set $\operatorname{Max} X$ is an antichain, and is therefore finite. Thus every downward closed set is the downward closure of its (finite) set of maximal elements.

Lemma 3.6. If $\boldsymbol{A}$ is an integral residuated ordered groupoid and $\boldsymbol{B}$ is a finite partial subalgebra of $\boldsymbol{A}$, then $\mathbf{D}(\boldsymbol{A}, \boldsymbol{B})$ is finite.

Proof. Let $B=\left\{b_{1}, \ldots, b_{k}\right\}$. Recall that $\mathbf{F}(k)$ is the free groupoid with identity 1 on $k$ generators $x_{1}, \ldots, x_{k}$. Let $\langle M, \cdot\rangle$ be the subgroupoid of $\boldsymbol{A}$ generated by $B$, as in Section 2. Then the map that sends $x_{i}$ to $b_{i}$ for each $i \in\{1, \ldots, k\}$ extends naturally to a surjective groupoid homomorphism $h: \mathbf{F}(k) \rightarrow\langle M, \cdot\rangle$.

Let $b \in B$ and consider the set $h^{-1}(b]=\{s \in \mathrm{F}(k): h(s) \leq b\}$, which is a subset of $\mathrm{F}(k)$. Denote by Crit $(b)$ the set of all maximal elements in $h^{-1}(b]$. As observed in the paragraph preceding this lemma, $\operatorname{Crit}(b)$ is a finite set, and since $h$ is order-preserving, $h^{-1}(b]=(\operatorname{Crit}(b)]^{\mathbf{F}}$. Set $Z=\bigcup_{b \in B} \bigcup_{z \in \operatorname{Crit}(b)}[z)^{\mathbf{F}}$, where $[z)^{\mathbf{F}}$ is the upward closure of $\{z\}$ in $\left\langle\mathrm{F}(k), \leq^{\mathbf{F}}\right\rangle$. It is evident from the definition of $\leq^{\mathbf{F}}$ that $[z)^{\mathbf{F}}$ is finite for each $z \in \mathrm{F}(k)$ since each larger term has fewer variables. (The fact also follows from the well-quasi-ordering of $\left\langle\mathrm{F}(k), \leq^{\mathbf{F}}\right\rangle$.) Thus, $B$, each $\operatorname{Crit}(b)$ and each $[z)^{\mathbf{F}}$ are finite, so $Z$ is finite.

Let $\boldsymbol{a}^{\boldsymbol{\delta}}=a_{1}^{\delta_{1}} \ldots a_{n}^{\delta_{n}} \in M^{l, r}$. Choose $u_{1}, \ldots, u_{n} \in \mathrm{F}(k)$ such that $h\left(u_{i}\right)=a_{i}$ for each $i \in\{1, \ldots, n\}$. We may write $\boldsymbol{a}^{\boldsymbol{\delta}}=h\left(u_{1}\right)^{\delta_{1}} \ldots h\left(u_{n}\right)^{\delta_{n}}$, which we abbreviate as $h\left(\boldsymbol{u}^{\boldsymbol{\delta}}\right)$. Thus, for $s \in \mathrm{F}(k), \boldsymbol{a}^{\boldsymbol{\delta}}(h(s))=h\left(\boldsymbol{u}^{\boldsymbol{\delta}}\right)(h(s))=h\left(\boldsymbol{u}^{\boldsymbol{\delta}}(s)\right)$ since $h$ is a groupoid homomorphism.

We show that $h^{-1}\left(\left(\boldsymbol{a}^{\boldsymbol{\delta}}\right)^{-1}(b]\right)=(Y]^{\mathbf{F}}$ for some $Y \subseteq Z$ :

$$
\begin{aligned}
s \in h^{-1}\left(\left(\boldsymbol{a}^{\boldsymbol{\delta}}\right)^{-1}(b]\right) & \Leftrightarrow h(s) \in\left(\boldsymbol{a}^{\boldsymbol{\delta}}\right)^{-1}(b] \\
& \Leftrightarrow \boldsymbol{a}^{\boldsymbol{\delta}}(h(s)) \leq b \\
& \Leftrightarrow h\left(\boldsymbol{u}^{\boldsymbol{\delta}}(s)\right) \leq b \\
& \Leftrightarrow \boldsymbol{u}^{\boldsymbol{\delta}}(s) \in h^{-1}(b] \\
& \Leftrightarrow \boldsymbol{u}^{\boldsymbol{\delta}}(s) \leq^{\mathbf{F}} t \quad \text { for some } t \in \operatorname{Crit}(b) .
\end{aligned}
$$

Using Lemma 3.3, we obtain

$$
\boldsymbol{u}^{\boldsymbol{\delta}}(s) \leq^{\mathbf{F}} t \quad \Leftrightarrow \quad s \leq^{\mathbf{F}} \rho\left(\boldsymbol{u}^{\boldsymbol{\delta}}\right)(t),
$$

hence

$$
s \in h^{-1}\left(\left(\boldsymbol{a}^{\boldsymbol{\delta}}\right)^{-1}(b]\right) \quad \Leftrightarrow \quad s \leq^{\mathbf{F}} \rho\left(\boldsymbol{u}^{\boldsymbol{\delta}}\right)(t) \quad \text { for some } t \in \operatorname{Crit}(b) .
$$


Set $Y=\left\{\rho\left(\boldsymbol{u}^{\boldsymbol{\delta}}\right)(t): t \in \operatorname{Crit}(b)\right\}$. Then $h^{-1}\left(\left(\boldsymbol{a}^{\boldsymbol{\delta}}\right)^{-1}(b]\right)=(Y]^{\mathbf{F}}$, the downward closure of $Y$ in $F(k)$, hence $\left(\boldsymbol{a}^{\boldsymbol{\delta}}\right)^{-1}(b]=h\left((Y]^{\mathbf{F}}\right)$, since $h$ maps $\mathrm{F}(k)$ onto $M$. For each $t \in \operatorname{Crit}(b), t \leq \mathbf{F} \rho\left(\boldsymbol{u}^{\boldsymbol{\delta}}\right)(t)$ (by Lemma 3.3)(ii)), hence $Y$ is a subset of $Z$. Thus each $\left(\boldsymbol{a}^{\boldsymbol{\delta}}\right)^{-1}(b] \in \bar{D}$ equals $h\left((X]^{\mathbf{F}}\right)$ for some $X \subseteq Z$.

Since $Z$ is finite, it has only finitely many subsets, hence there can be only finitely many distinct sets of the form $\left(\boldsymbol{a}^{\boldsymbol{\delta}}\right)^{-1}(b]$. This shows $\bar{D}$ is finite, and it follows that $\mathbf{D}(\boldsymbol{A}, \boldsymbol{B})$ is a finite algebra.

Theorem 3.7. The quasivariety IROG of integral residuated ordered groupoids has the FEP. Thus IROG is generated, as a quasivariety, by its finite members, and has a decidable universal theory.

By Theorem 2.7, we obtain

Corollary 3.8. For each $\mathcal{Q} \subseteq \mathcal{P}$, IROG ${ }^{\mathcal{Q}}$ has the FEP and hence is generated, as a quasivariety, by its finite members, and has a decidable universal theory.

In case the set of properties $\mathcal{Q}$ includes the associative law the description of $\mathbf{D}(\boldsymbol{A}, \boldsymbol{B})$ can be simplified. Indeed, if $\boldsymbol{A}$ is an associative residuated ordered groupoid and $\boldsymbol{B}$ is a partial subalgebra of $\boldsymbol{A}$, then, for $\boldsymbol{a}^{\boldsymbol{\delta}} \in M^{l, r}$ and $c \in M$, $\boldsymbol{a}^{\boldsymbol{\delta}}(c)$ simplifies to $a_{l} c a_{r}$, where $a_{l}$ is the product of all $a_{i}$ 's in $\boldsymbol{a}^{\boldsymbol{\delta}}$ that have an $l$ exponent and $a_{r}$ is the product of all $a_{i}$ 's in $\boldsymbol{a}^{\boldsymbol{\delta}}$ with an $r$ exponent. Associativity of multiplication allows us to remove all brackets. Thus,

$$
\left(\boldsymbol{a}^{\boldsymbol{\delta}}\right)^{-1}(b]=\left\{c \in M: a_{l} c a_{r} \leq b\right\} .
$$

When $\boldsymbol{A}$ is in addition commutative, we may further simplify $\left(\boldsymbol{a}^{\boldsymbol{\delta}}\right)^{-1}(b]$ to $\{c \in$ $\left.M: a_{l} a_{r} c \leq b\right\}$. Here, $a_{l} a_{r} \in M$, so writing $a_{l} a_{r}=a$, this set becomes $\{c \in M$ : $a c \leq b\}$ which we denoted by $(a \rightsquigarrow b]$ in [4]. The construction presented in this paper actually reduces to the one used in [4 for precisely the class of associative, commutative, integral residuated ordered groupoids.

\section{Failure of the FEP}

In the proof that IROG has the FEP, given in the previous section, the integrality condition plays an important role. We show here that if $\mathcal{Q} \subseteq \mathcal{P}$ contains the associativity condition but not the integrality condition, then $\mathrm{ROG}^{\mathcal{Q}}$ fails to have the FEP. The proof is a modification of the argument used in [4], where it is shown that the class of commutative, associative, unital residuated ordered groupoids does not have the FEP. We use ROM to denote the class of all associative residuated ordered groupoids (i.e., all residuated ordered semigroups), and for $\mathcal{Q} \subseteq \mathcal{P}$ we write $\mathrm{ROM}^{\mathcal{Q}}$ to denote the subclass of ROM satisfying all properties in $\mathcal{Q}$.

Let $\mathrm{Z}$ be the set of integers, + and - the usual addition and subtraction on $\mathrm{Z}$, and $\leq$ the usual linear order on $Z$. We add elements $\perp, T$, and extend $\leq$ to a linear order on $\mathrm{Z} \cup\{\perp, \top\}$ by setting $\perp<x<\top$ for all $x \in \mathrm{Z}$. We use $\perp$ and $T$ for the smallest and largest elements since 0 and 1 have a different meaning here. Next we extend the operation + over $\mathrm{Z} \cup\{\perp, \top\}$ by defining

$$
\begin{array}{ll}
\top+x=x+\top=\top \quad \text { if } x \neq \perp, \\
\perp+x=x+\perp=\perp & \text { for all } x .
\end{array}
$$


Then $\overline{\mathbf{Z}}=\langle\mathrm{Z} \cup\{\perp, \top\},+, \backslash, /, \leq\rangle$ is a residuated ordered groupoid with $x \backslash y=y-x$ for $x, y \in \mathrm{Z}$, and

$$
\begin{array}{ll}
x \backslash \top=\top \quad \text { for all } x, \\
\perp \backslash x=\top \quad \text { for all } x, \\
x \backslash \perp=\perp \quad \text { for all } x \neq \perp, \\
\top \backslash x=\perp \quad \text { for all } x \neq \top,
\end{array}
$$

and $y / x=x \backslash y$ for all $x, y$. In fact, $\overline{\mathbf{Z}}$ has all properties in the set $\mathcal{P}$ except for that of integrality: it is lattice ordered, upper and lower bounded, unital (with identity element 0 ), associative and commutative. However, $\overline{\mathbf{Z}}$ is not integral since $T \neq 0$.

Theorem 4.1. ROM does not have the FEP. For each $\mathcal{Q} \subseteq \mathcal{P}$ not containing the integrality property, $\mathrm{ROM}^{\mathcal{Q}}$ does not have the FEP.

Proof. Let $\varphi$ be the universal sentence

$$
\forall x, y\left[\left(x \leq x^{2} \& x^{2} y^{2} \approx x y\right) \Rightarrow\left(x y \approx x^{2} y\right)\right] .
$$

Then $\varphi$ fails in $\overline{\mathbf{Z}}$ since we may take $x=1$ and $y=-1$ and obtain $1 \leq 1+1$ and $1+1+(-1)+(-1)=1+(-1)$ but $1+(-1) \neq 1+1+(-1)$. Thus $\varphi$ fails in ROM and, in fact, $\varphi$ fails in each $\operatorname{ROM}^{\mathcal{Q}}$, where $\mathcal{Q} \subseteq \mathcal{P}$ does not contain the integrality condition.

We will show that $\varphi$ holds in every finite associative residuated ordered groupoid. Let $\boldsymbol{A}=\langle A, \cdot, \backslash, /, \leq\rangle \in \mathrm{ROM}$ be finite and let $a, b \in A$ such that $a \leq a^{2}$ and $a^{2} b^{2}=a b$. Since $a \leq a^{2}$, also $a^{k} \leq a^{k+1}$ for each $k<\omega, k \geq 1$, hence $a \leq a^{2} \leq$ $a^{3} \leq \ldots \leq a^{k} \leq a^{k+1} \leq \ldots$. Since $\boldsymbol{A}$ is finite, $a^{n}=a^{n+1}$ for some $n<\omega, n \geq 1$. Using $a^{2} b^{2}=a b$ to "cancel" middle terms, we have $a^{n} b^{n}=a \cdots a a b b \cdots b=a b$. Thus,

$$
a b=a^{n} b^{n}=a^{n+1} b^{n}=a\left(a^{n} b^{n}\right)=a a b=a^{2} b .
$$

This proves that $\varphi$ holds in $\boldsymbol{A}$. We have thus shown that ROM does not have the FEP.

Suppose $\mathcal{Q} \subset \mathcal{P}$ does not contain the integrality condition. Then $\overline{\mathbf{Z}} \in \mathrm{ROM}^{\mathcal{Q}}$, and hence the above argument shows that $\mathrm{ROM}^{\mathcal{Q}}$ fails to have the FEP as well.

Associativity is essential in the above argument and we have been unable to settle the following question.

\section{Problem 4.2. Does ROG have the FEP?}

The assumption of integrality has been crucial in our proofs that various classes of residuated ordered groupoids have the FEP, and it cannot be omitted even when we restrict ourselves to the class of commutative residuated lattice ordered monoids, according to Theorem 4.1. We conclude this section with a few results in which the assumption of integrality is replaced by $n$-potency.

For any class $\mathcal{K}$ of residuated ordered groupoids and $n \geq 1$, let $\mathcal{K}^{n}$ be the class of algebras in $\mathcal{K}$ satisfying the identity $x^{n} \approx x^{n+1}$, and let $\mathcal{K}_{\vee}$ be the class of members of $\mathcal{K}$ that are join-semilattices in the given order. Finally, CROS will denote the class of commutative residuated ordered semigroups.

Theorem 4.3. Let $1 \leq n<\omega$. The class $\mathrm{CROS}_{\vee}^{n}$ has the FEP. For each $n \geq 1$ and $\mathcal{Q} \subseteq \mathcal{P},\left(\mathrm{CROS}^{\mathcal{Q}}\right)_{\vee}^{n}$ has the FEP. 
Proof. Let $1 \leq n<\omega$. Let $\boldsymbol{A} \in \mathrm{CROS}_{\vee}^{n}$, and let $\boldsymbol{B}$ be a finite partial subalgebra of $\boldsymbol{A}$. We may assume that $\boldsymbol{A}$ has a least element 0, for if not, we may embed $\boldsymbol{A}$ into $\mathbf{D}(\boldsymbol{A}, \boldsymbol{A})$ (as in Corollary 2.8) which does have a least element, has a partial subalgebra isomorphic to $\boldsymbol{B}$, and belongs to $\mathrm{CROS}_{\vee}^{n}$ as well.

Let $\langle M, \cdot\rangle$ be the subsemigroup of $\boldsymbol{A}$ generated by the set $B \cup\{0,1\}$. Since $\langle M, \cdot\rangle$ is a finitely generated commutative, $n$-potent semigroup, $M$ is finite. In fact, $|M| \leq(n+1)^{|B|}+1$.

Let $N$ be the closure of $M$ under finite joins, i.e.,

$$
N=\left\{\bigvee_{i=1}^{m} c_{i}: m<\omega ; c_{1}, \ldots, c_{m} \in M\right\} .
$$

Observe that $N$ is closed under the groupoid operation of $\boldsymbol{A}$ since for $c_{1}, \ldots, c_{m}$, $d_{1}, \ldots, d_{l} \in N$, by (5) and (6),

$$
\left(\bigvee_{i=1}^{m} c_{i}\right)\left(\bigvee_{j=1}^{l} d_{j}\right)=\bigvee_{j=1}^{l}\left(\bigvee_{i=1}^{m} c_{i}\right) d_{j}=\bigvee_{j=1}^{l} \bigvee_{i=1}^{m} c_{i} d_{j}
$$

which is an element of $N$. Clearly, $N$ is also finite: we have $|N| \leq 2^{|M|}$. Since $N$ is closed under joins, we may define, for $a, b \in N$,

$$
a \backslash^{N} b=\bigvee\{c \in N: a c \leq b\} .
$$

Recall that $a 0=0 \leq b$ for all $a \in N$, so $\{c \in N: a c \leq b\}$ is never empty. For $a, b, c \in N$,

$$
a c \leq b \quad \Leftrightarrow \quad c \leq a \backslash^{N} b .
$$

The implication from left to right is immediate, and the implication from right to left follows by (31) and (5) of Section 1, For if $c \leq a \backslash^{N} b$, then

$$
a c \leq a\left(a \backslash^{N} b\right)=a(\bigvee\{c \in N: a c \leq b\})=\bigvee\{a c: c \in N ; a c \leq b\} \leq b .
$$

When $a, b \in B$ and $a \backslash b \in B$, (20) implies that $a \backslash^{N} b=a \backslash b$.

Since $\boldsymbol{A}$ is commutative, the operations / and $\backslash$ coincide, so we may define $/^{\boldsymbol{N}}=\backslash^{\boldsymbol{N}}$. Then, $\boldsymbol{N}=\left\langle N, \cdot, \backslash^{\boldsymbol{N}}, /^{\boldsymbol{N}}, \leq\right\rangle$ is a finite member of $\mathrm{CROS}_{\vee}^{n}$ and the identity map $\iota: \boldsymbol{B} \rightarrow \boldsymbol{N}$ an embedding. This shows that $\operatorname{CROS}_{\vee}^{n}$ has the FEP.

For the second part of the theorem, note that the finite algebra $\boldsymbol{N}$ already possesses all properties in $\mathcal{P}$ except perhaps that of being unital or integral. In particular, it is upper bounded since it is a finite join semilattice, and it is also a meet semilattice in the given order. For if we define, for $a, b \in N$,

$$
a \wedge^{N} b=\bigvee\{c \in N: c \leq a \wedge b\},
$$

then $\wedge^{N}$ is a meet operation on $N$ compatible with its order. Furthermore, if $1 \in B$, then $1=1^{N}$, and for $a, b \in B$, if $a \wedge b \in B$, then $a \wedge b=a \wedge^{N} b$, so 1 and $\wedge$ are preserved by the embedding.

If $\boldsymbol{A}$ is unital, then we can take $M$ to be the subsemigroup generated by $B \cup\{0, e\}$. This will ensure that $e \in N$, and hence that $\boldsymbol{N}$ is also unital. If in this case $\boldsymbol{A}$ is integral, i.e., if $e=1$, then the resulting algebra $N$ will clearly be integral as well.

The second statement of the last theorem was proved earlier in [4] under the assumption $\mathcal{Q}$ includes the integrality property.

In the last proof, commutativity was used to establish the finiteness of $M$. In the noncommutative case, if $n>1, M$ need not be finite. However, we do have the following. 
Theorem 4.4. $\mathrm{ROM}_{\vee}^{1}$ has the FEP, and more generally, for each $\mathcal{Q} \subseteq \mathcal{P},\left(\mathrm{ROM}^{\mathcal{Q}}\right)_{\vee}^{1}$ has the FEP.

Proof. Let $\boldsymbol{A} \in \mathrm{ROM}_{\vee}^{1}$ and let $\boldsymbol{B}$ be a finite partial subalgebra of $\boldsymbol{A}$. As before we may assume that $\boldsymbol{A}$ has a least element 0 . Let $\langle M, \cdot\rangle$ be the subgroupoid of $\boldsymbol{A}$ generated by the set $B \cup\{0\}$ and let $N$ be the closure of $M$ under finite joins, as in the proof of the previous theorem. Since $\langle M, \cdot\rangle$ is a finitely generated semigroup satisfying $x \approx x^{2}$, by a result of Green and Rees [11 $M$ is finite and hence $N$ is finite as well. Since $N$ is closed under · and $\bigvee$ we may define, for $a, b \in N$,

$$
\begin{aligned}
& a \backslash^{N_{b}}=\bigvee\{c \in N: a c \leq b\}, \\
& a /^{N_{b}}=\bigvee\{c \in N: c b \leq a\} .
\end{aligned}
$$

Then $\boldsymbol{N}=\left\langle N, \cdot \backslash^{\boldsymbol{N}},\left.\right|^{\boldsymbol{N}}, \leq\right\rangle$ is a finite member of $\mathrm{ROM}_{\vee}^{1}$ and $\boldsymbol{B}$ embeds into $\boldsymbol{N}$ by the identity map. This shows that $\mathrm{ROM}_{\vee}^{1}$ has the FEP. As before, all properties in $\mathcal{P}$ satisfied by $\boldsymbol{A}$ will hold in $\boldsymbol{N}$, and operations among $0,1, e, \wedge, \vee$ defined in $\boldsymbol{B}$ will be preserved by the embedding.

As the result by Green and Rees used in Theorem 4.4 does not extend to semigroups satisfying the law $x^{n} \approx x^{n+1}$ for $n>1$, we have the following problem.

Problem 4.5. Does $\mathrm{ROM}_{\vee}^{n}$ have the FEP if $1<n<\omega$ ?

Since Heyting algebras are 1-potent commutative residuated lattice ordered monoids, they are covered both by Theorem 4.3 and Theorem 4.4. McKinsey's and Tarski's well-known result that Heyting algebras have the FEP [15] follows thus from each as a special case.

\section{REFERENCES}

[1] G. Birkhoff, "Lattice Theory", Amer. Math. Soc., Providence, 1967. MR82a:06001

[2] W. J. Blok, D. Pigozzi, "Algebraizable logics", Memoirs of the American Mathematical Society, 396, Amer. Math. Soc., Providence, 1989. MR90d:03140

[3] W.J. Blok, J.G. Raftery, Varieties of commutative residuated integral pomonoids and their residuation subreducts, J. Algebra 190 (1997), 280-328. MR98j:08001

[4] W.J. Blok, C.J. van Alten, The finite embeddability property for residuated lattices, pocrims and BCK-algebras, Algebra Universalis 48 (2002), 253-271. MR2003j:06017

[5] T.S. Blyth, M.F. Janowitz, "Residuation Theory", Pergamon Press, Oxford-New York 1972. MR53:226

[6] Buszkowski, W., Mathematical linguistics and proof theory, in "Handbook of Logic and Language", J. van Benthem and A. ter Meulen, editors, North-Holland Publishing Co., Amsterdam; MIT Press, Cambridge, MA, 1997. MR98e:03025

[7] Dilworth, R. P, Non-commutative residuated lattices, Trans. Amer. Math. Soc. 46, (1939), 426-444. MR $1: 37 \mathrm{~d}$

[8] T. Evans, Some connections between residual finiteness, finite embeddability and the word problem, J. London Math. Soc. 1 (1969), 399-403. MR 40:2589

[9] I.M.A. Ferreirim, On varieties and quasivarieties of hoops and their reducts, Ph.D. thesis, University of Illinois at Chicago, 1992.

[10] L. Fuchs, Partially Ordered Algebraic Systems, Addison-Wesley, 1963. MR30:2090

[11] J. A. Green, D. Rees, On semi-groups in which $x^{r}=x$, Proc. Cambridge Philos. Soc. 48 (1952), 35-40. MR:13:720c

[12] G. Higman, Ordering by divisibility in abstract algebras, Proc. London Math. Soc. (3) 2 (1952) 326-336. MR14:238e

[13] D. Higgs, Dually residuated commutative monoids with identity element do not form an equational class, Math. Japon. 29 (1984), 69-75. MR86a:06021 
[14] Y. Lafont, The finite model property for various fragments of linear logic, J. Symbolic Logic 62 (1997), 1202-1208. MR99m:03121

[15] J.C.C. McKinsey, A. Tarski, On closed elements in closure algebras, Ann. of Math. 47 (1946), 122-162. MR7:359e

[16] M. Okada, K. Terui, The finite model property for various fragments of intuitionistic linear logic, J. Symbolic Logic 64 (1999) no. 2, 790-802. MR2002g:03043

[17] H. Ono, Y. Komori, Logics without the contraction rule, J. Symbolic Logic 50 (1985), 169202. MR 87a:03053

[18] C.J. van Alten, J.G. Raftery, On quasivariety semantics of fragments of intuitionistic propositional logic without exchange and contraction rules, Reports on Mathematical Logic 31 (1997), 3-55; also Internal Report No. 5/99, School of Mathematical and Statistical Sciences, University of Natal, Durban. MF2001e:03023

[19] Rosenthal, Kimmo I. "Quantales and their applications", Pitman Research Notes in Mathematics Series, 234. Longman Scientific \& Technical, Harlow; copublished in the United States with John Wiley \& Sons, Inc., New York, 1990. MR92e:06028

[20] M. Ward, R.P. Dilworth, Residuated lattices, Trans. Amer. Math. Soc. 45 (1939), 335-354.

[21] W. Wechler, "Universal Algebra for Computer Scientists", Springer-Verlag, BerlinHeidelberg, 1992. MR94a:08001

Department of Mathematics, Statistics and Computer Science, University of Illinois at Chicago, Chicago, Illinois 60607-7045

E-mail address: wjb@uic.edu

School of Mathematics, University of the Witwatersrand, Johannesburg, Wits 2050, South AFrica

E-mail address: cvalten@maths.wits.ac.za 